\title{
An attentional blink for sequentially presented targets: Evidence in favor of resource depletion accounts
}

\author{
Paul E. Dux, Christopher L. Asplund, And René Marois \\ Vanderbilt University, Nashville, Tennessee
}

\begin{abstract}
Several accounts of the attentional blink $(\mathrm{AB})$ have postulated that this dual-target deficit occurs because of limited-capacity attentional resources being devoted to processing the first target at the expense of the second (resource depletion accounts; e.g., Chun \& Potter, 1995). Recent accounts have challenged this model (e.g., Di Lollo, Kawahara, Ghorashi, \& Enns, 2005; Olivers, van der Stigchel, \& Hulleman, 2007), proposing instead that the $\mathrm{AB}$ occurs because of subjects' inability to maintain appropriate levels of attentional control when targets are separated by distractors. Accordingly, the AB is eliminated when three targets from the same attentional set are presented sequentially in a rapid serial visual presentation (RSVP) stream. However, under such conditions poorer identification of the first target is typically observed, hinting at a potential trade-off between the first and subsequent target performances. Consistent with this hypothesis, the present study shows that an $\mathrm{AB}$ is observed for successive targets from the same attentional set in an RSVP stream when the first target powerfully captures attention. These results suggest that resource depletion contributes significantly to the AB.
\end{abstract}

In rapid serial visual presentation (RSVP; Potter \& Levy, 1969), stimuli appear one after the other in the same spatial location for a fraction of a second each (e.g., $100 \mathrm{msec}$ ). Research using this method has identified a fundamental limitation in human conscious perception: the attentional blink (AB; Broadbent \& Broadbent, 1987; Raymond, Shapiro, \& Arnell, 1992; Weichselgartner \& Sperling, 1987), which refers to subjects' impaired ability to report the second of two targets in an RSVP stream if it appears within 200-600 msec of the first target.

A prominent account of the $\mathrm{AB}$ postulates that the phenomenon occurs because subjects devote limited attentional resources to the first target (T1) at the expense of the second (T2). According to this model, consolidation of T1 into working memory is attention demanding. If $\mathrm{T} 2$ is presented while $\mathrm{T} 1$ is being consolidated, as is thought to occur at short presentation lags between the two targets, $\mathrm{T} 2$ cannot be encoded and is susceptible to interference from the trailing distractor stimuli. At long temporal lags between T1 and T2, the first target is encoded into working memory before the presentation of the second target, and thus ample resources are available for $\mathrm{T} 2$ processing and the blink is avoided. This class of theories is often referred to as resource depletion (RD) accounts (e.g., Chun \& Potter, 1995; Jolicœur, 1998; Shapiro, Arnell, \& Raymond, 1997; Vogel, Luck, \& Shapiro, 1998) and localizes the AB effect to the limited-capacity stage of encoding information into working memory.

Recently, Di Lollo, Kawahara, Ghorashi, and Enns (2005; see also Kawahara, Enns, \& Di Lollo, 2006;
Kawahara, Kumada, \& Di Lollo, 2006; Olivers, van der Stigchel, \& Hulleman, 2007) have provided data that are seemingly at odds with RD models of the AB. In Di Lollo et al., subjects viewed RSVP streams of distractors that contained three successively presented targets. In one block of trials (uniform condition), all three targets were drawn from the same category (e.g., letters), and in the other (varied condition), the second target was drawn from a different category (e.g., computer symbols) than were the other two. A standard AB pattern was observed in the varied condition, with performance significantly impaired for the third target (T3) relative to the first. By contrast, no $\mathrm{AB}$ was observed in the uniform condition, despite the fact that $\mathrm{T} 3$ was presented at the same lag in both uniform and varied trials. These results were taken as evidence against $\mathrm{RD}$ models of the $\mathrm{AB}$ : According to $\mathrm{RD}$ accounts, an $\mathrm{AB}$ should also have been observed in the uniform condition because the same limited capacity resources would have been devoted to $\mathrm{T} 1$ processing in both uniform and varied conditions.

To account for these results and for the typical $A B$ observed in two-target RSVP search, several distractorbased theories of the deficit have been introduced that hypothesize that it is the stimulus directly following T1 (T1+1 stimulus), rather than $\mathrm{T} 1$ processing itself, that causes the AB. Di Lollo et al.'s (2005) temporary loss of control (TLC) hypothesis postulates that RSVP processing is governed by an input filter configured to select targets and exclude distractors. This filter is controlled 
by a central processor that can execute one operation at a time, and thus, in this model, it represents the bottleneck of information processing underlying the AB. TLC predicts that in two- or three-target RSVP, after the first target is identified, the central processor switches from stimulus monitoring to target consolidation, resulting in the input filter's being under exogenous control. When the stimulus immediately following T1 is another target from the same category as T1, the input filter's configuration is unaltered, and as a result, this target is also processed efficiently, eliciting lag 1 sparing (the absence of impaired performance on a second target when it appears directly after T1; see Visser, Bischof, \& Di Lollo, 1999). If, however, a stimulus drawn from a different category appears between $\mathrm{T} 1$ and $\mathrm{T} 2$, or if $\mathrm{T} 2$ is drawn from a different category from that for $\mathrm{T} 1$, processing of the second target is impaired because the input filter is no longer optimally configured for that target, thus causing the $\mathrm{AB}$.

Although Di Lollo et al. (2005) have proposed that impaired attentional control gives rise to the $\mathrm{AB}$, Olivers et al. (2007; see also Raymond et al., 1992) have hypothesized that a strengthening of control gives rise to the deficit. They suggested that the appearance of the $T 1+1$ distractor triggers a suppressive response. Because of the strength of this suppression, targets appearing in close proximity to $\mathrm{T} 1+1$ are also inhibited, resulting in impaired T2 performance at short lags relative to long lags. This model accounts not only for the AB found in standard dual-target RSVP paradigms but also for the absence of the deficit in paradigms employing sequential target presentation (uniform condition); with no suppression induced by the absence of a $\mathrm{T} 1+1$ distractor, performance on targets directly following $\mathrm{T} 1$ remains high.

The key difference between the $\mathrm{RD}$ and distractor-based accounts of the $\mathrm{AB}$ is that although the former class of theories suggests that the deficit is triggered by $\mathrm{T} 1$ processing, the latter predicts that the $\mathrm{AB}$ is the result of processes that occur following the presentation of the $\mathrm{T} 1+1$ distractor. On the surface, the complete attenuation of the $\mathrm{AB}$ in the uniform condition (in the three-target paradigm) is more easily explained by the distractor-based models than by RD theories. However, although such paradigms demonstrate much better T3 performance for the uniform than for the varied condition, they also often reveal the reversed pattern of performance for $\mathrm{T} 1$, with higher accuracy in varied trials than in uniform trials (e.g., Di Lollo et al., 2005; Kawahara, Enns, \& Di Lollo, 2006; Kawahara, Kumada, \& Di Lollo, 2006). Such a trade-off in target performance is not predicted by the distractor-based models, which postulate that $\mathrm{T} 1$ processing does not give rise to the $\mathrm{AB}$. By contrast, a trade-off in performance between the first and last targets is highly compatible with the $\mathrm{RD}$ accounts of the deficit.

Here we tested whether a trade-off in performance between $\mathrm{T} 1$ and $\mathrm{T} 3$ can account for the $\mathrm{AB}$ differences reported between the uniform and varied conditions in three-target RSVP paradigms. A key prediction of distractor-based theories is that the amount of resources devoted to processing $\mathrm{T} 1$ should not influence $\mathrm{T} 3$ performance under uniform conditions, because all the targets are drawn from the same attentional set, thereby leading neither to the reconfiguration of an input filter nor to distractor suppression. Put differently, if the $\mathrm{AB}$ is due to the appearance of a distractor immediately after $\mathrm{T} 1$, an $\mathrm{AB}$ should never be observed in uniform trials, because under these conditions, the $\mathrm{T} 1+1$ stimulus is a target of the same category as is T1. In two experiments, we manipulated the resources that subjects devoted to the first target and found that an $\mathrm{AB}$ can be observed in uniform trials if subjects increase the processing resources that they devote to T1. Our findings have strong implications for theories of the $\mathrm{AB}$, because they show that $\mathrm{T} 1$ resource depletion contributes to the deficit.

\section{EXPERIMENT 1}

Experiment 1 replicates the methodology of Di Lollo et al.'s (2005) second experiment, with subjects viewing RSVP streams of digit distractors that contained three targets presented successively. In the uniform condition, the targets were three letters; in the varied condition, $\mathrm{T} 1$ and $\mathrm{T} 3$ were letters and T2 was a digit (Figure 1). To manipulate the resources that subjects devoted to T1 processing, we varied, across groups, the color of the three target stimuli (as well as the color of posttarget distractors, in order to equate T3 masking). For one group of subjects, targets and distractors were both white, and for the other group, the targets (and the distractors that followed them) were red and the pretarget distractors were white. We reasoned that, with the target stimuli thus colored, T1 would capture attention because of its abrupt color onset (Maki \& Mebane, 2006), resulting in subjects' focusing more attentional resources on $\mathrm{T} 1$ processing. Indeed, research employing functional magnetic resonance imaging (fMRI) has demonstrated that abrupt color onset stimuli in RSVP elicit a larger blood-oxygen-level-dependent (BOLD) response in visual cortex than stimuli that do not represent abrupt color onsets (Serences et al., 2005). Thus, with T1 processing increased, we predicted decreases in $\mathrm{T} 3$ accuracy and therefore an $\mathrm{AB}$ in both the uniform and the varied conditions.

\section{Method}

Subjects. Twenty members ( 12 female and 8 male, mean age $=$ 20.5 years) of the Vanderbilt University community participated in the experiment. Half were allocated to the white target group and half were allocated to the red target group. All had normal or corrected-to-normal vision.

Design. Two independent variables, both of which had two levels, were manipulated: target color (white vs. red) and trial type (uniform vs. varied). Target color was manipulated between subjects, and trial type was manipulated within each group across blocks of trials. The dependent variables were T1, T2, and T3 report accuracy.

Stimuli and Apparatus. Stimuli were presented in Courier New font and subtended $1^{\circ}$ of visual angle at a viewing distance of approximately $57 \mathrm{~cm}$. Targets were three letters drawn from the entire alphabet (excluding I, L, O, Q, U, and V) in the uniform trials and were two letters and a digit (2-9) in the varied trials. Targets were colored red for one group of subjects and white for the other. The pretarget distractors were white digits for both groups and the posttarget distractors were of the same color as the targets, to ensure that the third target was always masked by a distractor of the same color and that only "letterness" defined targets for both groups (in the uniform condition). Distractors were also selected from the numbers $2-9$, and 


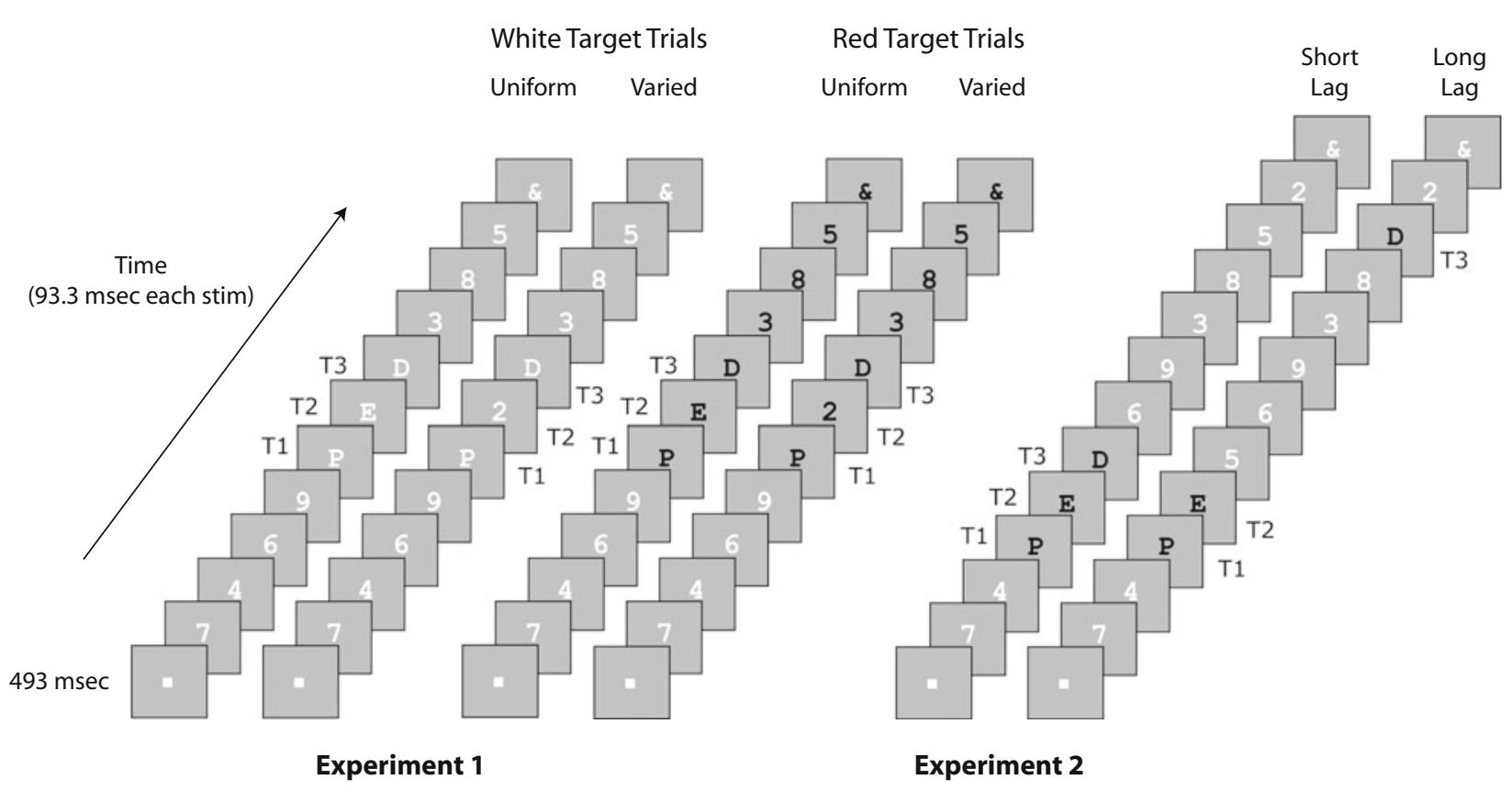

Figure 1. Example RSVP streams for Experiments 1 and 2. Black stimuli appeared in red in the experiments.

each stimulus appeared only once in each RSVP stream. Stimuli appeared centrally on a gray background for $93.3 \mathrm{msec}$ each with no interstimulus interval (ISI). The RSVP stream contained three targets, seven distractors, and an "\&" mask that appeared at the end of the stream. T1 appeared equally often at Serial Positions 3-7 and was immediately followed by $\mathrm{T} 2$ and $\mathrm{T} 3$. The experiment was programmed in MATLAB, using the Psychophysics Toolbox extension (Brainard, 1997; Pelli, 1997), and was conducted on an iMac computer with a $75-\mathrm{Hz}$ vertical refresh rate.

Procedure. Subjects initiated each trial by pressing the space bar. Trials began with a white fixation square for $493 \mathrm{msec}$, followed by the RSVP stream. Subjects performed either uniform or varied trials and entered the target identities, without time pressure, via a keyboard at the end of each RSVP stream when visually prompted (e.g., "Target 1?"). Written and verbal instructions that described the upcoming task and included diagrams of example RSVP streams were given to the subjects by the experimenter prior to study onset. For both groups, the instructions directed subjects to "identify all the targets with equal emphasis."

Each test block was made up of 120 trials, the first 20 of which were considered practice and were not analyzed. Half of the subjects in each group performed the varied block first; the other half performed the uniform block first.

\section{Results and Discussion}

Figure 2 shows the mean T1, T2, and T3 accuracy for uniform and varied trials plotted separately for each group. The data were submitted to a 2 (target color) $\times 2$ (trial type) $\times 3$ (target number) mixed-factorial ANOVA, which yielded a significant three-way interaction $[F(2,36)=3.7$, $p<.05$ ], indicating a different pattern of performance across the three targets in the two groups.

The results for the white target group replicate those reported by Di Lollo et al. (2005): There was a significant $\mathrm{AB}$ ( $\mathrm{T} 1$ accuracy $>\mathrm{T} 3$ accuracy) in the varied block $[t(9)=8.1, p<.0001]$, but not in the uniform block $(p=.3)$. In addition, T1 accuracy significantly differed between the uniform and varied trials, with $\mathrm{T} 1$ perfor- mance superior in the varied condition $[t(9)=4.7, p<$ $.005]$. This suggests a possible trade-off between $\mathrm{T} 1$ and T3 performance in the uniform block. Uniform and varied performance also differed significantly on the second target, with higher accuracy in the uniform trials than in the varied trials $[t(9)=11.8, p<.0001]$. This difference is found typically when one compares uniform and varied conditions (e.g., Di Lollo et al., 2005) and may reflect either a task-switch cost in the varied condition (report a digit as opposed to a letter) or a filtering cost resulting from T2 being drawn from the same category as that for the distractors.

Of primary interest was the pattern of results observed for the red target group. As was the case for the white target group, a significant AB (T1 accuracy $>\mathrm{T} 3$ accuracy) was observed in the varied condition $[t(9)=7.1$, $p<.0001]$, and T2 accuracy was higher in uniform trials than in varied trials $[t(9)=7.4, p<.0001]$. However, there was now also an $\mathrm{AB}$ observed in the uniform block $[t(9)=4.5, p<.005]$. In addition, T1 accuracy was greater for the red target group than for the white target group in both uniform $[t(9)=2.7, p<.02]$ and varied $[t(9)=2.7, p<.02]$ trials, and T3 was lower for the red target group than for the white target group in the uniform condition $[t(9)=2.2, p<.04]$, with a trend in the same direction for the varied condition $(p=.066)$. These results demonstrate that an $\mathrm{AB}$ can be observed under uniform conditions when the attentional resources devoted to T1 processing are increased. Taken together, these findings are consistent with the hypothesis that $\mathrm{T} 1$ resource depletion gives rise to the AB. They also suggest that the failure to observe the deficit in the white target uniform condition and previous three-target $\mathrm{AB}$ studies may have resulted from a trade-off in allocating processing resources between $\mathrm{T} 1$ and $\mathrm{T} 3$. 

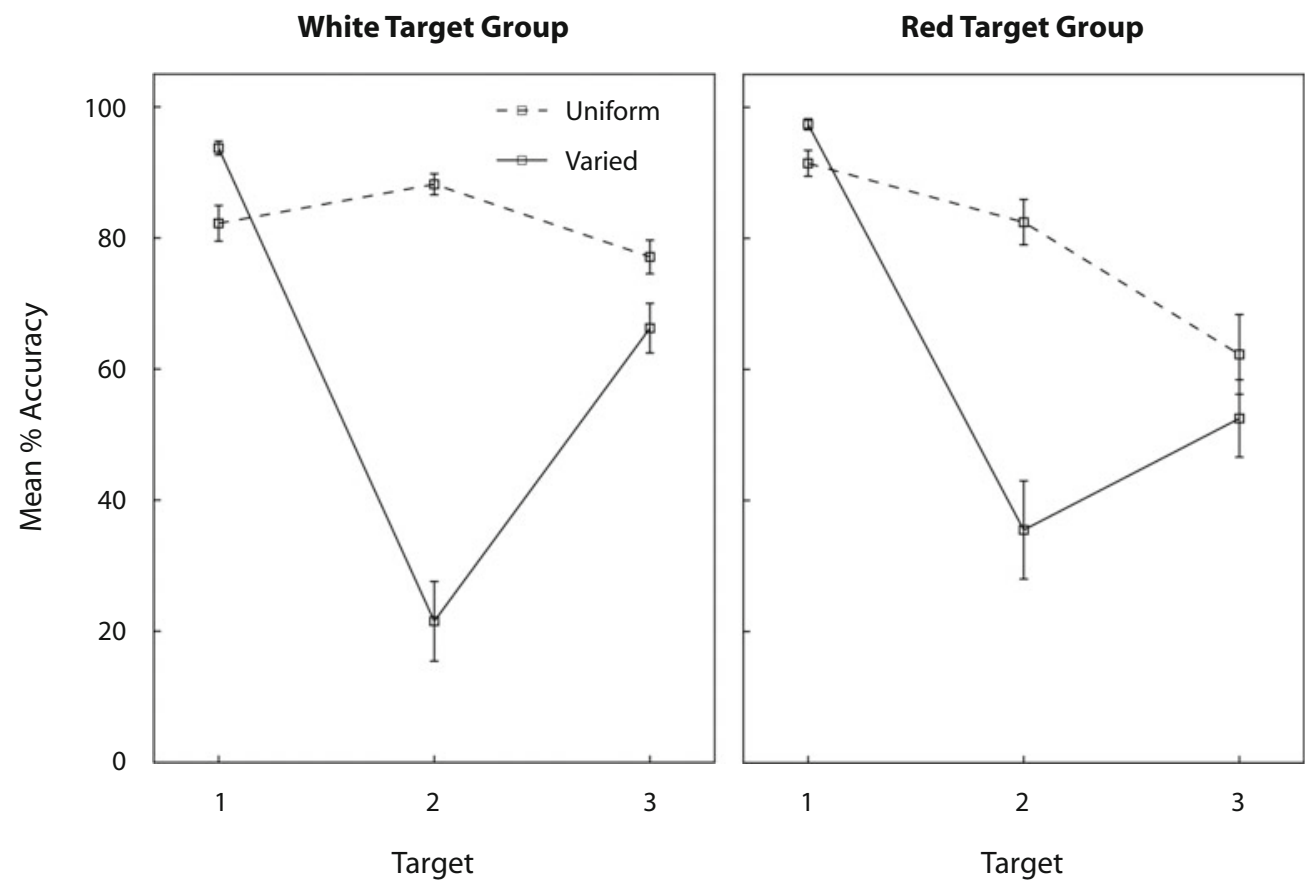

Figure 2. Mean T1, T2, and T3 accuracy in Experiment 1 as a function of trial type (uniform vs. varied), plotted separately for the white and red target groups.

\section{EXPERIMENT 2}

We have argued that an $\mathrm{AB}$ can be observed under uniform conditions if subjects increase the resources that they devote to T1 processing. However, in Experiment 1, we assessed the presence of an $\mathrm{AB}$ by contrasting performance on the first and third target (as has been done in previous three-target RSVP paradigms; see, e.g., Di Lollo et al., 2005), whereas typically, in dual-target RSVP paradigms, it is examined by manipulating lag between the targets (e.g., Chun \& Potter, 1995). To test whether the T3 deficit observed under uniform conditions for colored targets is indeed an $\mathrm{AB}$, we manipulated the $\mathrm{T} 1-\mathrm{T} 3$ lag in separate blocks of uniform trials, with the three targets appearing at either Serial Positions 3, 4, and 5 (short lag) or Serial Positions 3, 4, and 10 (long lag). If the T3 deficit observed for colored targets in uniform trials is an $\mathrm{AB}$, then $\mathrm{T} 3 \mathrm{ac}-$ curacy should be impaired in the short lag condition in comparison with the long lag condition, because only in the former was $\mathrm{T} 3$ presented while the other targets were still occupying the encoding bottleneck.

\section{Method}

The method for Experiment 2 was identical to that employed in the uniform red target condition of Experiment 1 except where specified.

Subjects. Ten subjects (including P.E.D. and C.L.A.; 4 female and 6 male, mean age $=21.6$ years) took part in Experiment 2 . The patterns of results for P.E.D. and C.L.A. were identical to those from the rest of the sample. Three additional subjects were excluded from the analysis because of low overall T1 accuracy ( $\leq 69 \%$; see Jolicœur, 1998).

Design. A single independent variable, T1-T3 lag (short lag and long lag), was manipulated across blocks of trials.
Stimuli and Apparatus. In both blocks of trials, three red letter targets appeared in an RSVP stream of eight white digits (see Figure 1). In the short-lag condition, the targets were presented at Serial Positions 3, 4, and 5; in the long-lag condition, the targets appeared at Serial Positions 3, 4, and 10.

Procedure. Subjects were instructed to give all targets equal emphasis and completed one block of each trial type. Each block consisted of 120 trials (the first 20 of which were practice), and their presentation order was counterbalanced across subjects.

\section{Results and Discussion}

Figure 3 plots mean T1, T2, and T3 performance in the short-lag and long-lag conditions. The data were submitted to a 2 (lag) $\times 3$ (target number) repeated measures ANOVA, which yielded a significant two-way interaction $[F(2,18)=7.7, p<.005]$, demonstrating that performance across the three targets was different in the two lag conditions.

Significant ABs were observed for both the shortlag trials $[t(9)=4.6, p<.005]$ and the long-lag trials $[t(9)=3.4, p<.01]$; however, the $\mathrm{AB}$ in the short-lag condition was significantly larger (over 2.5 times) than that observed in the long-lag condition $[t(9)=3.4, p<$ .01]. Importantly, these results cannot be due to forward masking differences between $\mathrm{T} 3$ in the short- and long-lag conditions (the former being forward masked by a red letter, the latter by a white digit), because $\mathrm{T} 2$ in the long-lag condition and T3 in the short-lag condition were masked identically yet performance on the stimuli was very different $[t(9)=5, p<.0001]$. Thus, the AB observed in the short-lag condition must be due to the temporal position of the third target. These results replicate Experiment 1 in showing that an $\mathrm{AB}$ can be produced under uniform conditions, and they further suggest that this $\mathrm{AB}$ is due, at 


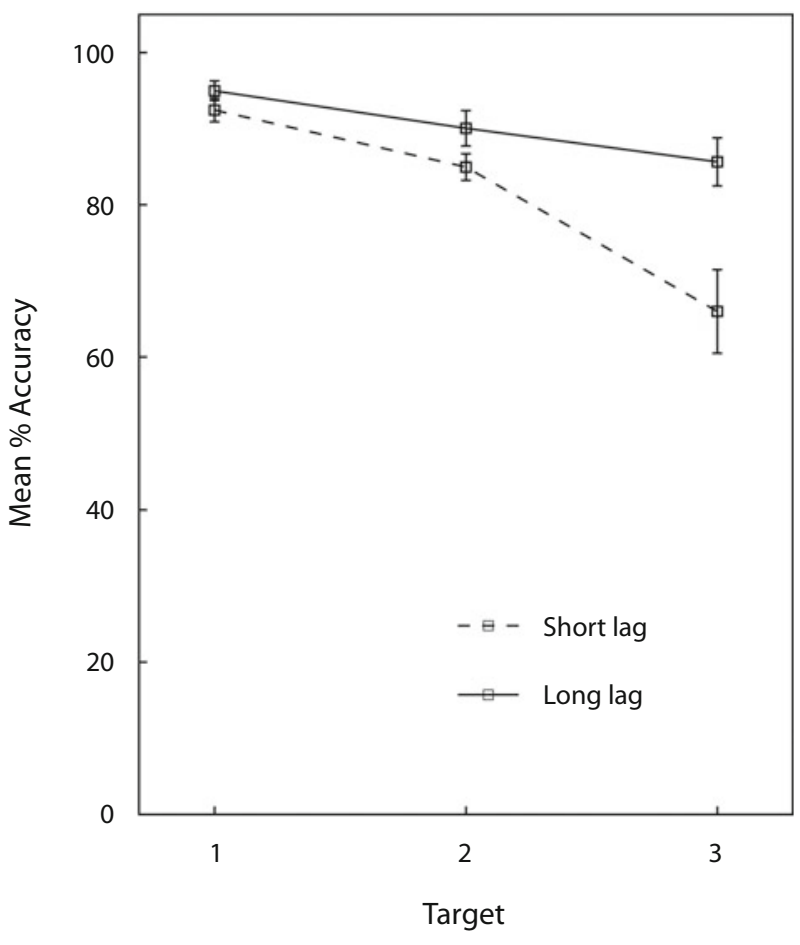

Figure 3. Mean T1, T2, and T3 accuracy in Experiment 2 as a function of T1-T3 lag (short lag vs. long lag).

least in part, to processing resources being devoted transiently to $\mathrm{T} 1$ at the expense of $\mathrm{T} 3$.

\section{CONCLUSION}

The present study contrasted RD and distractor-based theories of the AB. Previously, it has been demonstrated that an $\mathrm{AB}$ is not observed when three targets from the same attentional are presented successively in an RSVP stream (Di Lollo et al., 2005; Kawahara, Enns, \& Di Lollo, 2006; Kawahara, Kumada, \& Di Lollo, 2006; Olivers et al., 2007). This result has been taken as evidence that a T1+1 stimulus must disrupt an input filter (Di Lollo et al., 2005) or cause a strong suppressive response (Olivers et al., 2007) if an AB is to be elicited, and that $\mathrm{T} 1$ resource depletion does not give rise to the deficit. In the present study, we show that an $\mathrm{AB}$ can be observed even when all three targets belong to the same attentional set if subjects devote more resources to T1 processing, suggesting that previous failures to find an $\mathrm{AB}$ under these uniform conditions could have been due, at least in part, to subjects trading off performance between $\mathrm{T} 1$ and $\mathrm{T} 3$. Thus, the results presented here support the hypothesis that $\mathrm{T} 1$ resource depletion gives rise to the $\mathrm{AB}$ (e.g., Chun \& Potter, 1995; Jolicœur, 1998; Shapiro et al., 1997; Vogel et al., 1998) and demonstrate that a T1 processing bottleneck limits our ability to consciously perceive information that is distributed across time.

\section{AUTHOR NOTE}

This research was supported by NIMH Grant R01 MH70776 to R.M. We thank T. Megan Zoffuto for help with testing subjects. Correspondence concerning this article should be addressed to P. E. Dux, Department of Psychology, Vanderbilt University, 428 Wilson Hall, 111 21st Ave. So., Nashville, TN 37203 (e-mail: paul.dux@vanderbilt.edu).

\section{REFERENCES}

Brainard, D. H. (1997). The Psychophysics Toolbox. Spatial Vision, 10, 433-436.

Broadbent, D. E., \& Broadbent, M. H. P. (1987). From detection to identification: Response to multiple targets in rapid serial visual presentation. Perception \& Psychophysics, 42, 105-113.

Chun, M. M., \& PotTer, M. C. (1995). A two-stage model for multiple target detection in rapid serial visual presentation. Journal of Experimental Psychology: Human Perception \& Performance, 21, 109-127.

Di Lollo, V., Kawahara, J.-I., Ghorashi, S. M. S., \& Enns, J. T. (2005). The attentional blink: Resource depletion or temporary loss of control? Psychological Research, 69, 191-200.

JoliCGeUR, P. (1998). Modulation of the attentional blink by on-line response selection: Evidence from speeded and unspeeded Task ${ }_{1}$ decisions. Memory \& Cognition, 26, 1014-1032.

KaWAhara, J.-I., EnNS, J. T., \& Di Lollo, V. (2006). The attentional blink is not a unitary phenomenon. Psychological Research, 70, 405-413.

Kawahara, J.-I., Kumada, T., \& Di Lollo, V. (2006). The attentional blink is governed by a temporary loss of control. Psychonomic Bulletin \& Review, 13, 886-890.

MaKi, W. S., \& Mebane, M. W. (2006). Attentional capture triggers an attentional blink. Psychonomic Bulletin \& Review, 13, 125-131.

Olivers, C. N. L., van der Stigchel, S., \& Hulleman, J. (2007). Spreading the sparing: Against a limited-capacity account of the attentional blink. Psychological Research, 71, 126-139.

Pelli, D. G. (1997). The VideoToolbox software for visual psychophysics: Transforming numbers into movies. Spatial Vision, 10, 437-442.

Potter, M. C., \& LeVY, E. I. (1969). Recognition memory for a rapid sequence of pictures. Journal of Experimental Psychology, 81, 10-15.

Raymond, J. E., Shapiro, K. L., \& Arnell, K. M. (1992). Temporary suppression of visual processing in an RSVP task: An attentional blink? Journal of Experimental Psychology: Human Perception \& Performance, 18, 849-860.

Serences, J. T., Shomstein, S., Leber, A. B., Golay, X., Egeth, H. E., $\&$ YANTIS, S. (2005). Coordination of voluntary and stimulus-driven attentional control in human cortex. Psychological Science, 16, 114-122.

Shapiro, K. L., Arnell, K. M., \& Raymond, J. E. (1997). The attentional blink. Trends in Cognitive Sciences, 1, 291-296.

Visser, T. A. W., Bischof, W. F., \& Di Lollo, V. (1999). Attentional switching in spatial and nonspatial domains: Evidence from the attentional blink. Psychological Bulletin, 125, 458-469.

Vogel, E. K., LuCK, S. J., \& Shapiro, K. L. (1998). Electrophysiological evidence for a postperceptual locus of suppression during the attentional blink. Journal of Experimental Psychology: Human Perception \& Performance, 24, 1656-1674.

Weichselgartner, E., \& Sperling, G. (1987). Dynamics of automatic and controlled visual attention. Science, 238, 778-780.

(Manuscript received July 26, 2007;

revision accepted for publication February 22, 2008). 